\title{
Sensitivity analysis of the Künzel model: application to the study of the hygrothermal transfer in a tuffeau wall
}

\author{
I. Othmen $^{1,2}$, Ph. Poullain ${ }^{1}$, A. Caucheteux ${ }^{3} \&$ N. Leklou ${ }^{1}$ \\ ${ }^{1}$ Research Institute of Civil Engineering and Mechanics, \\ L'UNAM University, France \\ ${ }^{2}$ French Environment and Energy Management Agency, France \\ ${ }^{3}$ Centre for Studies and Expertise on Risks, Environment, Mobility, \\ and Urban and Country Planning, France
}

\begin{abstract}
The concerns regarding sustainable development bring to light the importance of the consumption of natural energy resources by the building sector. The thermal renovation of old buildings should be a priority for reducing greenhouse gas emission. In fact, tuffeau, the main stone of Loire Valley, France, is a very porous limestone $(43 \%)$ and seems to be very sensitive to atmospheric conditions. So, an inappropriate insulation technique can lead to moisture disorders harmful to the sustainability of the wall. Its compatibility with an insulation material can be numerically studied using a combined heat and moisture transfer model. One of the most important aspects on modeling is the knowledge of the material properties. The numerical tool WUFI based on Künzel model leads to identify the parameters whose measurement effort must be intensified as a result of a local sensitivity analysis. Those parameters are adsorption isotherm and convective coefficients in the case of static conditions. Keywords: tuffeau, renovation, hygrothermal performance, WUFI, sensitive analysis.
\end{abstract}

\section{Introduction}

The energy consumption related to the building sector is recognized as the major consumption in the EU. According to the Eurosat statistics in 2012 as nearly $40 \%$ of final energy consumption, and consequently a significant source of 
greenhouse gas emissions happen at houses. In France, around $70 \%$ of this energy is used for heating a partially ageing building stock $(66 \%$ of the residential stock was built before 1974) [1]. Many efforts have to be done to insulate and thermally improve these buildings. In this context, professionals need to assess insulation techniques for old buildings made of tuffeau. Tuffeau is the main building stone of castles and old houses in the Loire valley, France. The walls made of this highly porous stone are prone to heat and moisture transfers. The implementation of a classical insulation technique could lead to disturbance of the hygrothermal transfer through the wall and then deteriorate significantly its performances.

A numerical tool based on hygrothermal approach is therefore essential in order to examine the risk of hygrothermal disturbance related to an insulation technique. However, heat and moisture transfer models require the knowledge of material related parameters and boundary conditions. Some of them can easily be measured but some others require great efforts to achieve reliable values. Nevertheless, it may be useless to seek high accuracy of a parameter if the heat and moisture transfer model is weakly sensitive to this parameter.

In the present study, the Künzel model is used to evaluate the influence of input parameters on its main outputs. Sensitivity analysis (SA) can allow to identify parameters for which a reduction in uncertainty specification will have the most significant impact on improving model performance measures or on which parameters the precision effort could be reduced. SA has been used in previous studies for this purpose and for various applications from finance to life sciences, from quantum physics to earth sciences and environmental engineering [2-4]. There are many different SA approaches. Overall, they can be categorized into two groups: local SA and global SA. The local SA explores the changes of model response by varying one parameter while keeping other parameters constant. However the global SA examines the changes of model response by varying all parameters at the same time.

\section{Methods and material}

\subsection{Heat and moisture transfer model}

In the past two decades, numerical simulation has drawn a growing attention as being an efficient technique to study hygrothermal performance of building envelopes. Several numerical tools to predict combined heat, air, and moisture transport in building envelopes have been quoted in various publications [5-8]. Significant differences between them exist, such as the choice of driven potential of moisture transport, transfer mechanisms included in the conservation equations, the material properties needed as input data or the boundary conditions.

In the present work, we used the heat and moisture transfer model developed by Künzel [8, 9]. The governing equations are heat and mass balance equations, expressed as follows: 


$$
\left\{\begin{array}{c}
\rho_{0} C_{p} \frac{\partial T}{\partial t}=\frac{\partial}{\partial x}\left(\lambda \frac{\partial T}{\partial x}\right)+L_{v} \frac{\partial}{\partial x}\left(\delta_{P} \frac{\partial \varphi P_{s a t}}{\partial x}\right) \\
\frac{\partial w}{\partial \varphi} \frac{\partial \varphi}{\partial t}=\frac{\partial}{\partial x}\left(D_{\varphi} \frac{\partial \varphi}{\partial x}\right)+\frac{\partial}{\partial x}\left(\delta_{P} \frac{\partial \varphi P_{\text {sat }}}{\partial x}\right)
\end{array}\right.
$$

The first equation deals with heat transfer by temperature gradient, driven thermal conduction and convection of latent heat of vaporisation. The second equation represents the moisture transfer due to relative humidity and water vapour pressure gradients. Both equations contain storage (volumetric heat capacity $\rho_{0} C_{p}$ and adsorption isotherm $\frac{\partial w}{\partial \varphi}$ ) and transport terms (thermal conductivity $\lambda$, liquid diffusivity $D_{\varphi}$ and water vapour permeability $\delta_{p}$ ), which all should depend on the material temperature and/or water content. The transport in liquid phase is controlled by the moisture gradient . The transport coefficient $D_{\varphi}$ increases significantly with the water content while the influence of the temperature is limited to its effect on the viscosity of water. Vapor diffusion is governed by the water vapour partial pressure which depends on the relative humidity and the saturation water vapour pressure $P_{\text {sat }}$. The diffusion coefficient $\delta_{p}$ depends slightly on the temperature and the water content while the saturation vapour pressure increases exponentially with temperature. The thermal conductivity $\lambda$ and the specific heat $C_{p}$ both depend on water content.

We carried out the simulations with the commercial software WUFI ${ }^{\circledR}$ developed at Fraunhofer institute for building physics (IBP) and based on the discretisation of the Künzel model by means of an implicit finite volume scheme.

\subsection{Material}

The tested wall is made of tuffeau. It is a stone composed of two main minerals: calcite $\mathrm{CaCO}_{3}$ and silica $\mathrm{SiO}_{2}$. Silica is shared between the quartz, opals and clay minerals. While calcite is present in two sizes: sparitic calcite $(\varnothing \approx 100 \mu \mathrm{m})$ and micritic calcite $(\varnothing<10 \mu \mathrm{m})$ [11]. The latter forms with opals the stone cement. The properties that served as the model input parameters are given in table 1 .

\subsection{Wall description}

The wall geometry is described on figure 2. We tested the wall under static conditions by maintaining constant temperature and relative humidity on the inner and outer wall surfaces (table 2). The convection heat transfer coefficients for both inner and outer wall are set constant and their values are attributed according to the standard (ISO 6946). We set the lower and upper boundaries perfectly insulated and impermeable to ensure unidirectional heat and moisture transfer along the $x$ axis. 
Table 1: The basic data of the tuffeau.

\begin{tabular}{|c|c|c|}
\hline Parameter & & Value \\
\hline Dry bulk density, $\rho_{0}$ & 1310 & {$\left[\mathrm{~kg} / \mathrm{m}^{3}\right]$} \\
\hline Porosity, $n$ & 0.487 & {$\left[\mathrm{~m}^{3} / \mathrm{m}^{3}\right]$} \\
\hline Dry specific heat, $C_{p}$ & 1000 & {$[\mathrm{~J} / \mathrm{kg} . \mathrm{K}]$} \\
\hline Dry thermal conductivity,$\lambda$ & 0.41 & {$\left[\mathrm{~W} / \mathrm{m}^{2} \cdot \mathrm{K}\right]$} \\
\hline Water vapor diffusion resistance factor, $\mu$ & 30 & {$[-]$} \\
\hline Water absorption coefficient,$A$ & 0.5 & {$\left[\mathrm{~kg} / \mathrm{m}^{2} \cdot \mathrm{s}^{0.5}\right]$} \\
\hline \multicolumn{3}{|c|}{$\begin{array}{l}\text { Parameters of the GAB model, expressed below, for the adsorption isotherm } \\
\text { (figure 1) }\end{array}$} \\
\hline$w=w_{m} \frac{k \cdot C \cdot \varphi}{(1-k \varphi)(1-k(1-C) \varphi)}$ & & (2) \\
\hline $\begin{array}{l}w_{m}: \text { monolayer water content } \\
\mathrm{C}: \text { fitting parameter } \\
\mathrm{k}: \text { fitting parameter }\end{array}$ & $\begin{array}{l}0.082 \\
14.1289 \\
0.9329\end{array}$ & $\begin{array}{l}{\left[\mathrm{kg} / \mathrm{m}^{3}\right]} \\
-\end{array}$ \\
\hline
\end{tabular}

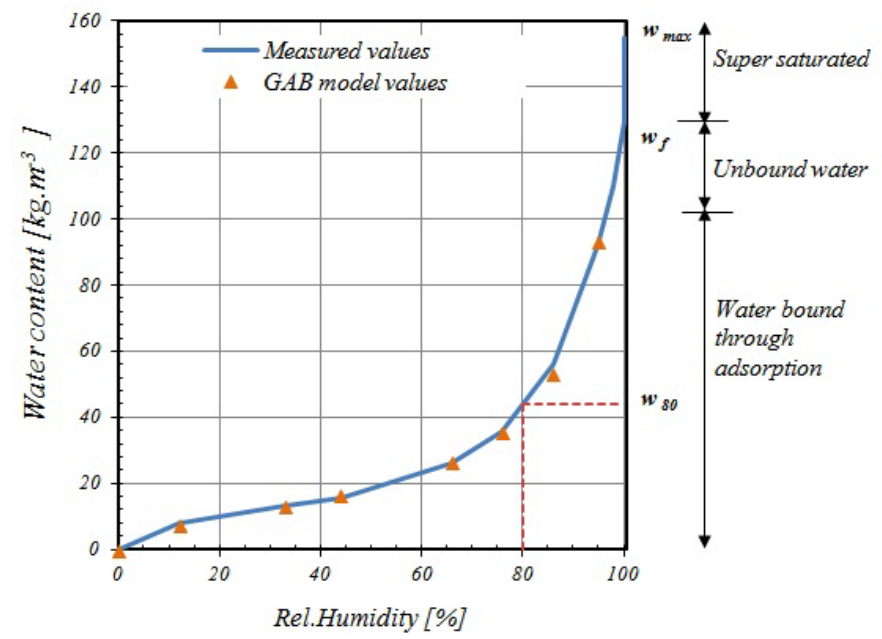

Figure 1: The adsorption isotherm of tuffeau.

Table 2: Boundary conditions and moisture source.

\begin{tabular}{|c|c|c|c|c|c|c|c|}
\hline \multirow{2}{*}{ Duration } & \multicolumn{2}{|c|}{$\begin{array}{c}\text { Initial } \\
\text { conditions }\end{array}$} & \multicolumn{2}{c|}{$\begin{array}{c}\text { Inner } \\
\text { conditions }\end{array}$} & \multicolumn{2}{c|}{$\begin{array}{c}\text { Outer } \\
\text { conditions }\end{array}$} & $\begin{array}{c}\text { Moisture } \\
\text { source }\end{array}$ \\
\hline \multirow{3}{*}{$3000 \mathrm{~h}$} & $T\left({ }^{\circ} \mathrm{C}\right)$ & $\varphi(\%)$ & $T\left({ }^{\circ} \mathrm{C}\right)$ & $\varphi(\%)$ & $T\left({ }^{\circ} \mathrm{C}\right)$ & $\varphi(\%)$ & $\begin{array}{c}\text { No } \\
\text { capillary } \\
\text { rise }\end{array}$ \\
\cline { 2 - 7 } & 20 & 50 & 20 & 50 & 5 & 90 & \\
\hline
\end{tabular}



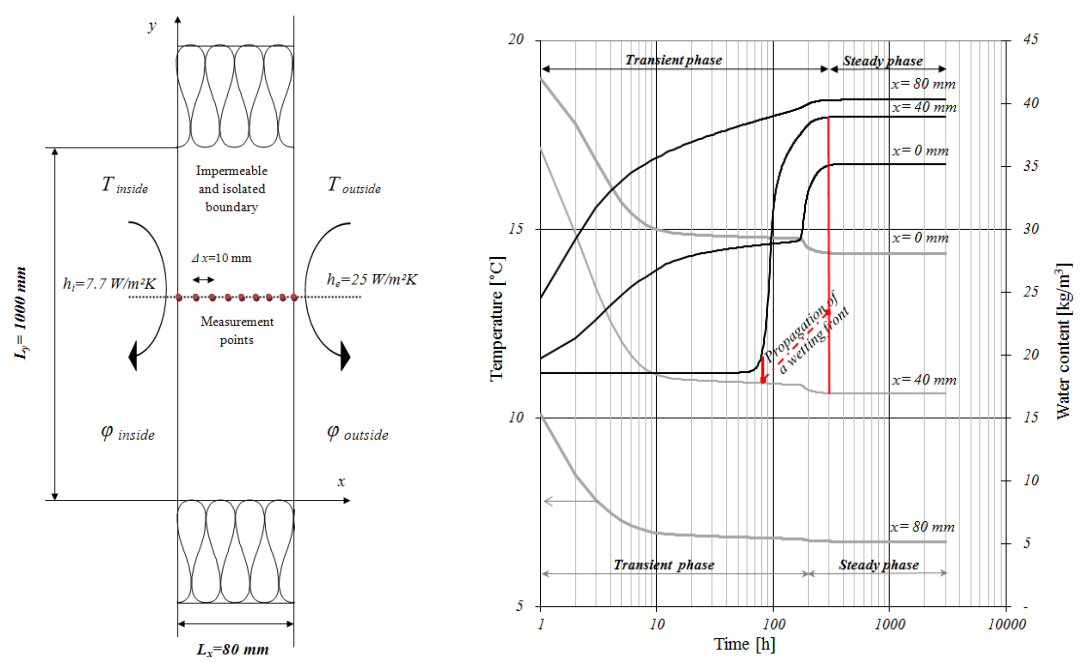

Figure 2: $\quad$ Tested wall (left) - typical time evolution of the temperature and water content in the wall as calculated by the Künzel model with parameters given in tables 1 and 2 (right).

\subsection{Sensitivity indicators}

The parameters of the model exert great influence on model performance $[2,12]$. Some of the parameters may be observed or measured thus they can be vitiated by an error.

As previously pointed out, the values of the model input parameters are known with uncertainty. The goal of this paper is to quantify the influence of this uncertainty on the wall hygrothermal evolution. We applied a local sensitivity analysis (LSA) to point out the parameters that have an impact on the main model outputs: temperature and water content at different positions in the wall. To achieve the analysis, the parameters were varied in a $\pm 5 \%$ range around the reference material parameter value and $\pm 10 \%$ around the convection heat transfer coefficients. We carried out 19 simulations varying 9 parameters and assuming that these parameters can be varied independently.

We selected the relative error $\delta_{y}$ as an indicator of sensitivity:

$$
\delta y(\%)=100 \frac{\left|y_{\text {sim }}-y_{\text {ref }}\right|}{y_{\text {ref }}}
$$

where $\delta_{y}$ is the relative error, $y_{\text {sim }}$ is the output value determined with varied parameter $(\Delta= \pm 5 \%$ or $\Delta= \pm 10 \%)$ and $y_{\text {ref }}$ is the output value without uncertainty $(\Delta=0 \%)$.

We calculated the relative error $\delta_{y}$ from temperature, relative humidity and water content time evolution during the transient phase, defined as the time necessary to achieve $99 \%$ of the whole field variation between the initial and the 
steady states. Indeed, as the boundary conditions are static, we observe a transient phase followed by a steady phase, as shown on figure 2 .

We performed statistical study of the relative errors for the different parameters and presented the results by means of the "Box and Whiskers Plot" or "Quartile Boxes" (figure 3). The box contains quartiles and includes an additional character to represent the mean of the data. The ends of the whiskers represent the minimum $(m)$ and maximum $(M)$ of all of the data.

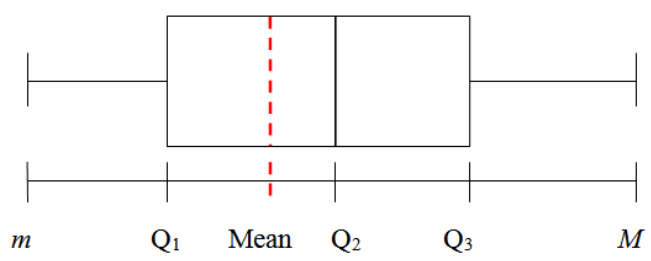

Figure 3: An example of Box plot with whiskers.

- The first quartile $Q_{l}$ is the lower quartile and it means that $25 \%$ of the data are lower than this value.

- The second quartile $Q_{2}$ is also called the median and it divides the data set in two equal populations.

- The third quartile $Q_{3}$ is the upper quartile and it represents $75 \%$ of the data.

\section{Results}

We obtained the results presented in this section by performing 19 simulations and varying 9 parameters.

To illustrate the method of statistical analysis, we chose to present the results concerning the influence of the inner convection heat transfer coefficient on the temperature time evolution and of the adsorption isotherm on the water content time evolution. We thus selected a material parameter and a boundary parameter.

\subsection{Raw data comparison}

Figure 4 presents the temperature and water content time evolution at five different positions in the wall: $0 \mathrm{~mm}$ (inner wall surface), $20 \mathrm{~mm}, 40 \mathrm{~mm}, 60 \mathrm{~mm}$ and $80 \mathrm{~mm}$ (outer wall surface). Groups of curves are related to the same position and different values of the considered parameter (reference value and upper and lower value). The change in a parameter value can modify the temperature or water content time evolution, but the global curve shape remains unchanged.

For the temperature, each curve at a defined position starts from the same temperature because the varied parameter refers to the inner boundary condition. 
The initial state is the same and the difference between the curves increases with time. A lower value of $h_{i}$ yields a lower temperature profile, which is in accordance with theoretical conclusions related to the influence of this parameter. Furthermore, the influence of $h_{i}$ vanishes as the distance from the inner surface increases. The inner heat transfer coefficient $h_{i}$, as well as $h_{o}$, has thus a global and local influence on the temperature time evolution. In fact, $h_{i}$ impacts the heat flow from the indoor air to the surface. The same conclusions were made in studies $[12,13]$.

For the water content, differences appear right from the start because the initial condition is defined as an initial relative humidity. A variation in the adsorption isotherm logically implies differences in the initial water content. The adsorption isotherm exhibiting higher values of the water content for a given relative humidity yields higher curves. Unlike the boundary parameter $h_{i}$, a change in a material related parameter implies changes in a field time evolution of the same order of magnitude over the whole wall. The effect of the change in the adsorption isotherm has thus a global effect on the material evolution.

These observations from raw data are qualitative remarks that need to be completed with quantitative analysis.
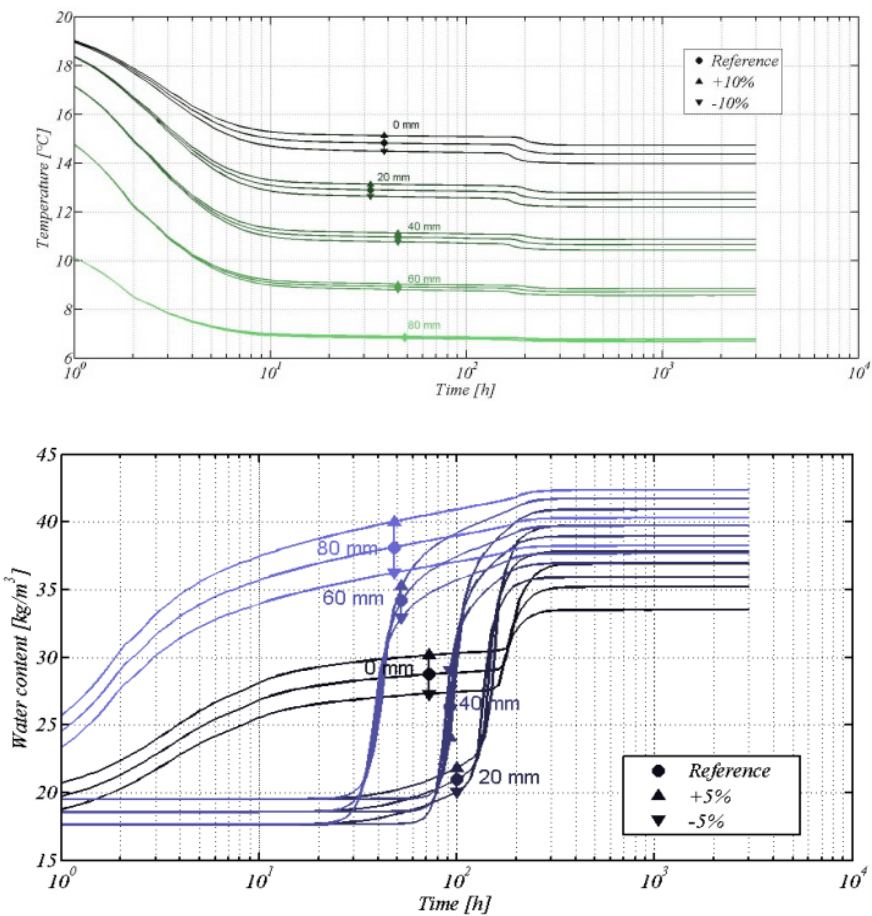

Figure 4: $\quad$ Time evolution of the temperature for $h_{i}$ (top) and of water content for adsorption isotherm (bottom). 


\subsection{Statistical study}

We analysed the differences between the reference curve and the curves with varied parameters by means of local or global computation of statistical indicators. The density probability functions are computed and presented in the form of quartile boxes. Figure 5 and 6 present the local quartile boxes determined respectively from the temperature time evolution with variable $h_{i}$ and from the water content time evolution with variable adsorption isotherm.

We can see on figure 5 that the influence of $h_{i}$ is more important at the inner surface and decreases with depth. The repartition around the median (quartile $Q_{2}$ ) is tight as compared to the relative error range. For example, at $10 \mathrm{~mm}$ the relative error ranges from $0 \%$ to $3 \%$ for $h_{i}+10 \%$ whereas $Q_{1}$ is about $2 \%$ and $Q_{3}$ less than $2.1 \%$. We conclude that at $10 \mathrm{~mm}$, the relative error is quite constant during the transient phase. These observations confirm those made in the previous section. Another important piece of information is that an uncertainty of $10 \%$ on the value of $h_{i}$ yields a difference of only $2.5 \%$ on the temperature profile.

The quartile boxes for the adsorption isotherm (figure 6), underline the global influence of this parameter on the water content. The impact is equal to the uncertainty initially introduced $( \pm 5 \%)$ and is nearly constant throughout the wall except for surfaces $(\mathrm{x}=0 \mathrm{~mm}$ and $\mathrm{x}=80 \mathrm{~mm})$. We can explain it by the importance of the boundary conditions at the surfaces compared to the properties of materials. This graph shows that accurately measuring the adsorption isotherm is of great importance to simulate the water content evolution in a wall, because the uncertainty is not reduced by the model.

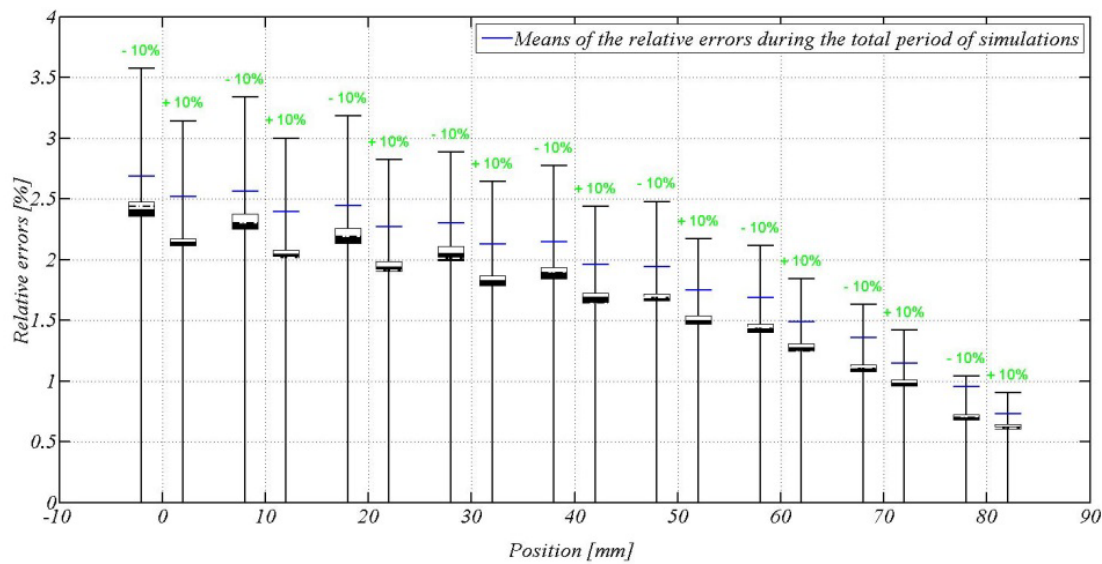

Figure 5: Evolution of the relative errors for temperature due to $h_{\text {i variability. }}$. 


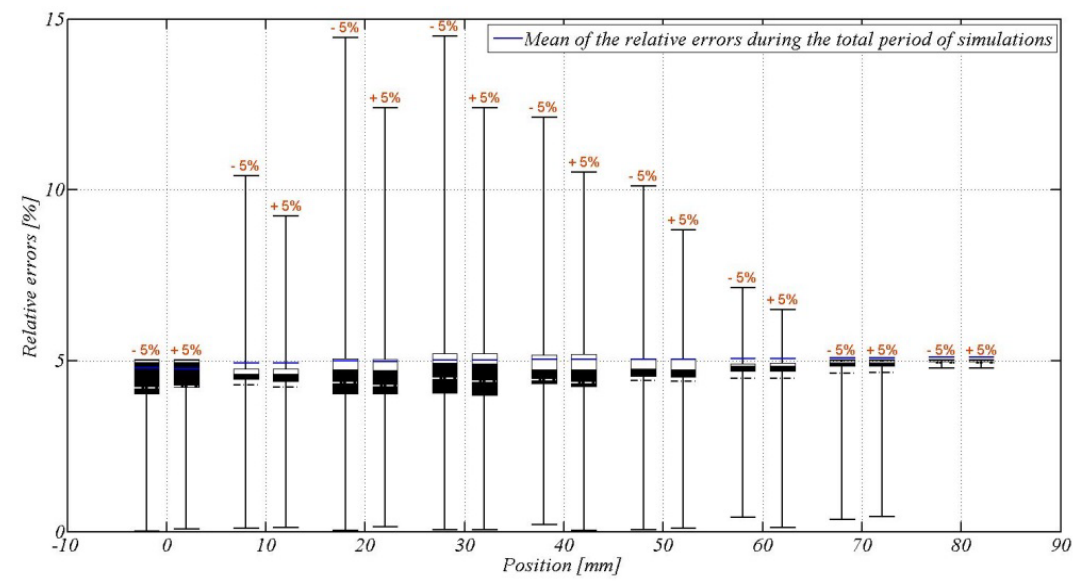

Figure 6: Evolution of the relative errors for water content due to adsorption isotherm variability.

In order to classify the parameters with respect to their influence on the temperature or water content fields, we computed the statistical indicators over the whole wall thickness. Figure 7 and 8 present the results for the 9 parameters.

The less influent parameters are obviously the porosity $n$ and the resistance to water vapour diffusion $\mu$. Even with uncertainty of $5 \%$, the mean relative error remains lower than $0.1 \%$. In this particular case of heat and moisture transfer through a tuffeau wall, these parameters do not have to be accurately determined. The same observation can be done for the density $\rho$ and the specific heat capacity $C_{p}$, for which the mean relative error is lower than $0.1 \%$, even if the total range is larger (1\%). The Künzel model is not very sensitive to uncertainty on these parameter values.

We propose a classification for the sensitivity of the Künzel model towards the parameters of the model (table 3 ).

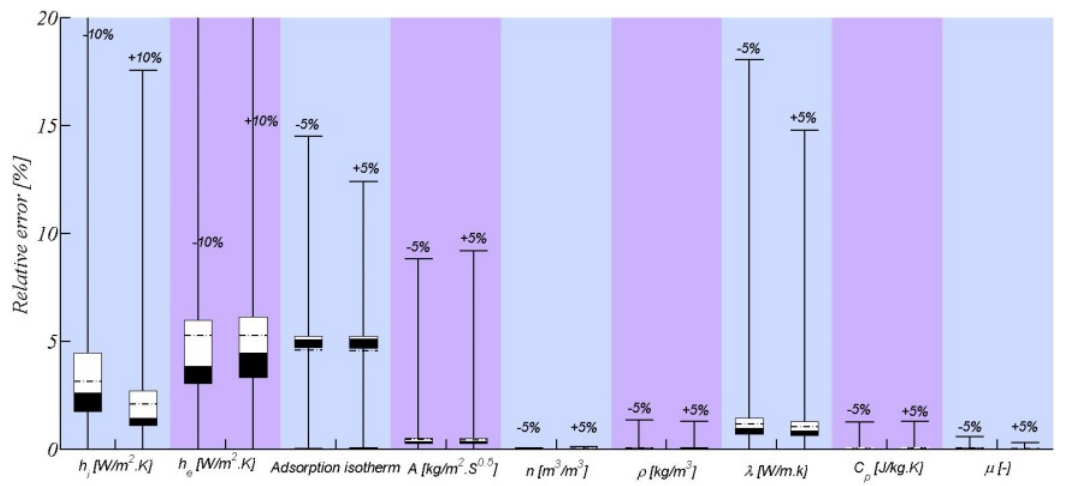

Figure 7: Evolution of the relative errors for water content. 


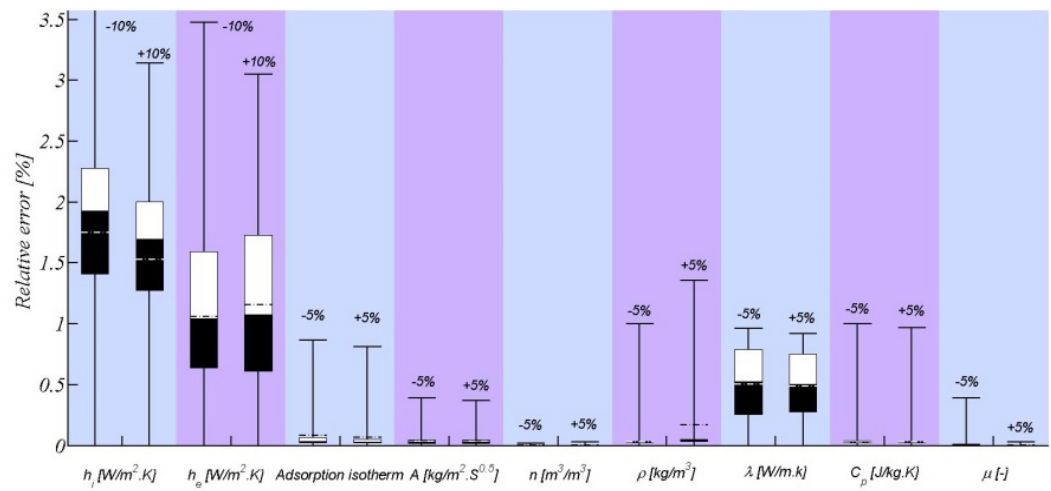

Figure 8: Evolution of the relative errors for the temperature.

Table 3: Classification of the Künzel model parameters.

\begin{tabular}{|c|c|c|}
\hline \multirow{2}{*}{ Sensitivity } & \multicolumn{2}{|c|}{ Parameter } \\
\cline { 2 - 3 } & Temperature & Water content \\
\hline Low & $w(\varphi), A, n, \mu, \rho, C_{p}$ & $n, \mu, \rho, C_{p}$ \\
\hline Medium & $\lambda$ & $A, \lambda$ \\
\hline High & $h_{i,}, h_{o}$ & $w(\varphi), h_{i,} h_{o}$ \\
\hline
\end{tabular}

\subsection{Discussion}

We carried out the simulations on a single layer wall. The same simulation on a multilayer wall could lead to a different classification, especially for the thermal conductivity and the volumetric heat capacity, or more generally for the diffusion coefficient and the storage parameters.

Moreover, the results obtained in this study are relative to a $80 \mathrm{~mm}$ thick wall and for the tuffeau. In future works, it is necessary to achieve more general results, in particular by taking into account the relative influence of the different parameter values. Indeed, different time scale can be associated to the material properties: thermal time scale, moisture diffusion time scale, water vapour transfer time scale... We can build dimensionless numbers with these different time scales, which characterize the predominant phenomena in the hygrothermal evolution of the wall. The tuffeau is characterized by its own dimensionless numbers and the classification we proposed could be only relative to this particular case as pointed out in [14] and [15]. It could thus be necessary to perform simulations on the basis of dimensionless numbers.

\section{Conclusion}

The local sensitivity analysis in case of a wall under static conditions allows defining influential parameters which are the adsorption isotherm and the convection heat transfer coefficients. Those parameters play an important role in 
the water content of the material. The temperature profile is also sensitive to the variations of the convective coefficients.

Nevertheless, the results we presented could be relative to a single layer tuffeau wall. Future work should be dedicated to the determination of the influence of the material properties through dimensionless parameters to avoid this limitation.

\section{Acknowledgement}

This work is supported by the French Agency for Environment and Energy (ADEME) and Nantes University.

\section{References}

[1] RAGE 2012, Analyse détaillée du parc résidentiel existant (September 2012), report published January 2013.

[2] D. Garcia Sanchez, B. Lacarrière, M. Musyb, B. Bourgesaa: Application of sensitivity analysis in building energy simulations: Combining first- and second-order elementary effects methods, Energy and Buildings Volume 68, Part C, 2014, Pages 741-750.

[3] Xu. M, Yang. J, Gao. Z: Parameters Sensitive Analyses for Using Genetic Algorithm to Solve Continuous Network Design Problems, Procedia Social and Behavioral Sciences, Volume 43,2012, Pages 435-444.

[4] Burch D.M., J. Chi :MOIST, A PC Program for Predicting Heat and Moisture Transfer in Building Envelopes, Release 3.0, NIST Special Publication, 917 National Institute of Standards and technology, Gaithersburg (1997).

[5] Mendes. N, P.C. Philippi, R. Lamberts: A new mathematical method to solve highly coupled equations of heat and mass transfer in porous media, International Journal of Heat and Mass Transfer, Volume 45, Issue 3, January 2002, Pages 509-518.

[6] Woloszyn. M, C. Rode: Tools for performance simulation of heat, air and moisture conditions of whole building. Building simulation (2008) 1: 5-24.

[7] Qinru .L, J. Rao, P. Fazio: Development of HAM tool for building envelope analysis, Building and Environment, Volume 44, Issue 5, May 2009, Pages 1065-1073.

[8] Künzel. H.M: Simultaneous Heat and Moisture Transport in Building Components. - One- and two-dimensional calculation using simple parameters. IRB Verlag (1995).

[9] Künzel. H.M: Effect of interior and exterior insulation on the hygrothermal behavior of exposed walls. Materials and Structures 31 (1998), H. 206, Pages 99-103.

[10] Beck. K, Al-Mukhtar. M, Rozenbaum. O, Rautureau .M: Characterization, water transfer properties and deterioration in tuffeau: building material in 
the Loire valley - France, , Building and Environment, Volume 38, Issue 9-10, Septemer-October 2003, Pages 1151-1162.

[11] Spitz. C, Mora. L, Wurtz. E: Simulating combined heat and moisture transfer with energy plus: an uncertainty study and comparison with experimental data, Building simulation conference, Chambéry, France, August 25-28, 2013.

[12] Hamad. M.A.A., Uddin. Md. J, A.I. Md. Ismail: Investigation of combined heat and mass transfer by Lie group analysis with variable diffusivity taking into account hydrodynamic slip and thermal convective boundary conditions, International Journal of Heat and Mass Transfer, Volume 55, 2012, Pages 1355-1362.

[13] Andreas. H.H, Künzel. H.M: Practical application of an uncertainty approach for hygrothermal building simulations - drying of an AAC flat roof, Building and environment, Volume 37, Issue 8, Pages 883-889.

[14] Defraeye. T, Blocken. B, J. Carmeliet: Influence of uncertainty in heat-moisture transport properties on convective drying of porous materials by numerical modeling, Chemical Engineering Research and Design, Volume 91, Issue 1, Pages 36-42. 